\title{
Die „Finanzthemen“ im Reformprozess: Kommissionsauftrag, Optionen, politische Diskussion
}

Nachdem die zum 1. September 2006 in Kraft getretene „Föderalismusreform I“ vor allem eine Entflechtung der Gesetzgebungszuständigkeiten von Bund und Ländern zum Gegenstand hatte, nahmen Bundestag und Bundesrat im Dezember desselben Jahres die „Zweite Stufe“ der Reform in Angriff, die sich den bundesstaatlichen Finanzbeziehungen widmen soll. Dies umfasst etwa Fragen einer Reform des Steuerverbundes, aber auch mögliche Regelungen zur Begrenzung der staatlichen Kreditaufnahme und zum Umgang mit der aufgelaufenen Verschuldung der Gebietskörperschaften. Hinzu treten verwaltungsbezogene, aber dennoch finanzwirksame Fragen wie die einer Aufgabenkritik, einer materiellen Entbürokratisierung und einer Effizienzsteigerung des öffentlichen Handelns. Die nachfolgende Dokumentation gibt einen Überblick über den derzeitigen Stand der Kommissionsarbeiten zu den sog. „Finanzthemen“"1 und informiert in diesem Zusammenhang über Einsetzung und Auftrag der Kommission (I.), die seitens der Sachverständigen vorgetragenen Reformoptionen (II.) sowie die in diesem Rahmen geführte politische Diskussion (III.). Ein kurzer Ausblick (IV.) beschließt die Darstellung.

\section{Einsetzung und Auftrag der „Föderalismuskommission II“}

Nach dem Vorbild der ersten Reformstufe wurde erneut eine gemeinsame Kommission eingesetzt, die aus je 16 Mitgliedern des Bundestages und Vertretern des Bundesrates bestand. Hinzu kamen ohne Stimmrecht vier Vertreter der Landesparlamente und die Präsidenten der drei Kommunalen Spitzenverbände. ${ }^{2}$ Den

1 Auf die Verwaltungsthemen wird in dieser Dokumentation nicht näher eingegangen. Vgl. dazu die Beiträge in der Rubrik Abhandlungen dieses Heftes.

2 Deutscher Bundestag, 16. Wahlperiode: Einsetzung einer gemeinsamen Kommission zur Modernisierung der Bund-Länder-Finanzbeziehungen, BT-Drs. 16/3885 vom 14. 12. 2006; gleichlautend Bundesrat: Einsetzung einer gemeinsamen Kommission zur Modernisierung der Bund-Länder-Finanzbeziehungen, BR-Drs. 913/06 (Beschluss) vom 15. 12. 2006. 
Vorsitz übernahmen gemeinsam der Vorsitzende der SPD-Bundestagsfraktion, Struck, und der baden-württembergische Ministerpräsident Oettinger (CDU). ${ }^{3}$

Gemäß den Einsetzungsbeschlüssen sollte die Kommission Vorschläge zur Reform der Bund-Länder-Finanzbeziehungen erarbeiten „,mit dem Ziel, diese den veränderten Rahmenbedingungen inner- und außerhalb Deutschlands insbeson-

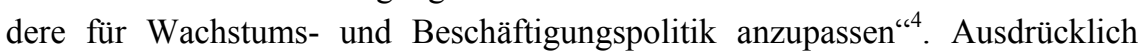
genannt wurden die Ziele einer verstärkten Eigenverantwortung der Gebietskörperschaften und einer aufgabenadäquaten Finanzausstattung. Die den Beschlüssen beigefügte „offene Themensammlung“ nannte darüber hinaus die Vorbeugung von und die Bewältigung bestehender Haushaltskrisen, Aufgabenkritik und Standardsetzung, Entbürokratisierung und Effizienzsteigerung, verstärkte $\mathrm{Zu}$ sammenarbeit und freiwillige Zusammenschlüsse von Ländern sowie die Bündelung fachpolitischer Leistungen. ${ }^{5}$

\section{Reformoptionen für die bundesstaatliche Finanzverfassung: Die Positionen der Sachverständigen}

Die Kommission unterteilte ihren Auftrag in zwei inhaltliche Blöcke: „Finanzthemen " sowie „Verwaltungsthemen “. Zu beiden wurde je eine umfangreiche Anhörung externer Sachverständiger abgehalten. ${ }^{6}$ Mit Blick auf die Finanzthemen richtete die Kommission vorab einen Katalog von insgesamt 226 Fragen an die Sachverständigen, der sich in die Teile „Haushalt und Schulden“ sowie „Einnahmen und Kompetenzen" gliederte. Ersterem kam dabei mit 150 Fragen ein zumindest quantitativ - deutliches Übergewicht zu. ${ }^{7}$ Die Auswahl der Sachverständigen für die öffentliche Anhörung zu den Finanzthemen am 22. Juni 2007 erfolgte über die verschiedenen „Bänke“ der Kommission (Bundestagsfraktio-

3 Kommission von Bundestag und Bundesrat zur Modernisierung der Bund-Länder-Finanzbeziehungen: Komm.-Prot. 01 vom 08. 03. 2007, 3.

4 Deutscher Bundestag, 16. Wahlperiode, a.a. O., 1; Bundesrat, a.a. O., 1.

5 Deutscher Bundestag, 16. Wahlperiode, a.a. O., Anlage 1; Bundesrat, a. a. O., Anlage 1.

6 Kommission von Bundestag und Bundesrat zur Modernisierung der Bund-Länder-Finanzbeziehungen: Komm.-Prot. 04 vom 22.06. 2007 (Finanzthemen); dies.: Komm.-Prot. 08 vom 08.11. 2007 (Verwaltungsthemen).

7 Kommission von Bundestag und Bundesrat zur Modernisierung der Bund-Länder-Finanzbeziehungen: Fragenkatalog für die öffentliche Anhörung zu den Finanzthemen am Freitag, dem 22. Juni 2007, KDrs. 011.; vgl. zu den Schwerpunkten der Kommissionsarbeit auch Baus, R. T./Margedant, U.: Stand der Föderalismusreform und Auftrag der Föderalismuskommission II, in: Baus, R.T./Eppler, A./Wintermann, O. (Hg.): Zur Reform der föderalen Finanzverfassung in Deutschland, Baden-Baden, 2008, 13-29, hier $19 \mathrm{ff}$. 
nen, Bundesrat, Landtage, Kommunale Spitzenverbände), hinzu kamen zwei Vertreter des Sachverständigenrates zur Begutachtung der gesamtwirtschaftlichen Entwicklung. ${ }^{8}$ Im Folgenden werden die Positionen zu den Schlüsselproblemen in komprimierter Form dargelegt.

\section{Rechtliche Vorkehrungen zur Begrenzung der Neuverschuldung}

Mit Blick auf die Frage, ob und inwieweit Kreditaufnahme durch die öffentliche Hand zu ermöglichen oder zu unterbinden sei, gingen die Meinungen überwiegend dahin, dass Verschuldung nicht per se problematisch sei. Es komme vielmehr darauf an, welchen Zwecken sie diene und wie die Tilgungsmodalitäten ausgestaltet seien. Zudem sei eine an der wirtschaftlichen Leistungsfähigkeit orientierte Begrenzung der Neuverschuldung sinnvoll. ${ }^{9}$ Ein absolutes Verschuldungsverbot wurde hingegen überwiegend abgelehnt. ${ }^{10}$

Unter den Gründen, die öffentliche Kreditaufnahme rechtfertigen könnten, wurden insbesondere genannt:

- Wirkung der automatischen Stabilisatoren in Phasen eines konjunkturellen Abschwungs, Verhinderung prozyklischer Fiskalpolitik; ${ }^{11}$

- längerfristige Glättung von staatlichen Einnahmen und Ausgaben zur Vermeidung gesamtwirtschaftlicher Wohlfahrtsverluste (tax-smoothing-Theorie); ${ }^{12}$

8 Kommission von Bundestag und Bundesrat zur Modernisierung der Bund-Länder-Finanzbeziehungen: Komm.-Prot. 04 vom 22.06. 2007. Eine Übersicht aller teilnehmenden Sachverständigen mit Ausweis der jeweils benennenden „Bank“ findet sich dort auf S. VII ff.

9 Junkernheinrich, M.: Stellungnahme zur Öffentlichen Anhörung der Kommission von Bundestag und Bundesrat zur Modernisierung der Bund-Länder-Finanzbeziehungen am 22. Juni 2007, K-Drs. 034, 24.

10 Korioth, S.: Stellungnahme zur öffentlichen Anhörung der Kommission von Bundestag und Bundesrat zur Modernisierung der Bund-Länder-Finanzbeziehungen am 22. Juni 2007, K-Drs. 017 vom 24.05. 2007, 6; Renzsch, $W$ : Stellungnahme zur Anhörung am 22. Juni 2007, K-Drs. 016 vom 24. 05. 2007, 5; Seitz, H.: Stellungnahme zum Fragenkatalog für die öffentliche Anhörung zu den Finanzthemen am Freitag, dem 22. Juni 2007, der Kommission von Bundestag und Bundesrat zur Modernisierung der Bund-Länder-Finanzbeziehungen, K-Drs. 023, Mai 2007, 44. Für eine strikte Regelung jedoch Meyer, H.: Stellungnahme zum Fragenkatalog der Föderalismuskommission II (Kommissionsdrucksache 11), K-Drs. 014 vom 18.05. 2007, 12. Vgl. im Übrigen den Beitrag von Joachim Wieland in diesem Heft.

11 Häde, U.: Schriftliche Stellungnahme zur Vorbereitung der öffentlichen Anhörung der Kommission von Bundestag und Bundesrat zur Modernisierung der Bund-Länder-Finanzbeziehungen am 22. Juni 2007 in Berlin, K-Drs. 021 vom 25.05.2007, $10 \mathrm{f}$; Huber, B.: Modernisierung der Bund-LänderFinanzbeziehungen. Eine ökonomische Analyse, K-Drs. 018, Mai 2007, 2.

12 Fuest, C.: Stellungnahme zum Fragenkatalog der Kommission von Bundestag und Bundesrat zur Modernisierung der Bund-Länder-Finanzbeziehungen (Kommissionsdrucksache 11) anlässlich der öf- 
- Vorhaltung risikofreier Anlagemöglichkeiten für private Wirtschaftssubjekte ${ }^{13}$

- Beteiligung künftiger Generationen an der Finanzierung von Investitionen, gerechtfertigt durch entsprechenden Nutzen für diese, ${ }^{14}$

- Bereitstellung finanziellen Spielraums für den Staat im Fall außergewöhnlicher Ereignisse (etwa Naturkatastrophen). ${ }^{15}$

Entgegengehalten wurden dem vor allem die folgenden Argumente:

- zunehmender Zweifel an staatlicher Steuerungsfähigkeit der Konjunktur in einer globalisierten Welt; ${ }^{16}$

- Ablehnung einer Sonderstellung für Investitionen unter den staatlichen Aufgaben, Recht zur Erhebung von Zwangsabgaben auch bei diesen ausreichend zur Finanzierung; ${ }^{17}$

- noch stärkere Einschränkung politischer Gestaltungsmöglichkeiten durch Verpflichtung zum Schuldendienst. ${ }^{18}$

Ungeachtet dieser Positionen bestand Einigkeit, dass die öffentliche Verschuldung in der Bundesrepublik Deutschland in den vergangenen Jahrzehnten ein zu großes Ausmaß angenommen habe. Zur Frage nach den Ursachen wurden nicht nur exogene Ereignisse und politische Entscheidungen bzw. deren Ausbleiben (etwa bei der Reform des Sozialstaates) angeführt, sondern auch unzureichende Vorkehrungen in der Finanzverfassung. Dies bezog sich auf die „Schuldenbremse“ in Art. 115 Abs. 1 S. 2 GG, die derzeit wie folgt gefasst ist: „Die Einnahmen

fentlichen Anhörung zu den Finanzthemen am 22. Juni 2007, K-Drs. 019 vom 25.05.2007, 9; Huber, B., a.a.O., 2.

13 Kirchgässner, G.: Stellungnahme zum Fragenkatalog der Kommission von Bundestag und Bundesrat zur Modernisierung der Bund-Länder Finanzbeziehungen, K-Drs. 027 vom 27. 05. 2007, 8.

14 Huber, B., a.a.O., 2; Sachverständigenrat zur Begutachtung der gesamtwirtschaftlichen Entwicklung: Schriftliche Stellungnahme zum Fragenkatalog für die Anhörung am 22. Juni 2007, K-Drs. 033 vom 12.06. 2007, 2.

15 Junkernheinrich, M., a.a.O., 36; Lenk, T.: Stellungnahme zum Fragenkatalog der Föderalismuskommission II, K-Drs. 026, 10.

16 Feld, L.P.: Schriftliche Stellungnahme für die Anhörung der Föderalismuskommission II zu den Finanzthemen am 22. Juni 2007, K-Drs. 024 vom 28. 05. 2007, 27; Meyer, H., a. a. O., 12.

17 Meyer, H., a.a.O., 12.

18 Ebd., 9; Konrad, K. A.: Stellungnahme zur öffentlichen Anhörung der Kommission von Bundestag und Bundesrat zur Modernisierung der Bund-Länder-Finanzbeziehungen am 22. Juni 2007, K-Drs. 020 vom 25.05. 2007, 3; Schneider, H.P.: Gestaltungsföderalismus statt Verwaltungsföderalismus Stellungnahme zur öffentlichen Anhörung der Kommission von Bundestag und Bundesrat zur Modernisierung der Bund-Länder-Finanzbeziehungen am 22. Juni 2007 in Berlin, K-Drs. 031, Juni 2007, 18. 
aus Krediten dürfen die Summe der im Haushaltsplan veranschlagten Ausgaben für Investitionen nicht überschreiten; Ausnahmen sind nur zulässig zur Abwehr einer Störung des gesamtwirtschaftlichen Gleichgewichts.“ Ähnliche Regelungen finden sich in den meisten Länderverfassungen. ${ }^{19}$

Diese Begrenzung der staatlichen Befugnis zur Kreditaufnahme wurde allgemein als nicht ausreichend kritisiert. ${ }^{20}$ So sei etwa die auf eine nicht näher konkretisierte „Störung des gesamtwirtschaftlichen Gleichgewichts“ abstellende Ausnahmeregelung zu offen formuliert und werde daher zu häufig in Anspruch genommen. Zudem werde der Investitionsbegriff auf Bruttoinvestitionen bezogen. Dadurch, dass etwa Abschreibungen und Desinvestitionen (insbesondere Privatisierungen) bei der Berechnung des zulässigen Verschuldungsspielraums unberücksichtigt blieben, erhöhe sich dieser zusätzlich. ${ }^{21}$ Hinzu komme schließlich, dass die Regelungen auch insofern sehr „weich“ seien, als sie auch bei offensichtlichen Verstößen entweder überhaupt keine Sanktionen vorsähen oder diese nur mit sehr großer Verzögerung wirksam würden. ${ }^{22}$ Eine abweichende Auffassung vertrat der Sachverständige Wieland: Demnach seien vor allem eine lang andauernde ungünstige Wirtschaftslage sowie politische Fehlentscheidungen ursächlich für die Zunahme der Staatsverschuldung. Die Steuerungswirkung der Finanzverfassung sei bei der Festlegung einer Verschuldungsregel grundsätzlich begrenzt, sie wirke dabei eher als politischer Appell denn als zwingendes Recht. $^{23}$

Auch weitere systemimmanente Anreizstrukturen begünstigten eine Zunahme der Verschuldung. Insbesondere sei es politisch einfacher, Kredite aufzunehmen, als die durch sie finanzierten Ausgaben zu kürzen. ${ }^{24}$ Des Weiteren verwies der Sachverständige Junkernheinrich auf die kaum vorhandene persönliche Verantwortlichkeit der entscheidenden Akteure. ${ }^{25}$

19 Fuest, C., a. a. O., 6.

20 Blankart, C. B.: Wer soll für die Schulden von Bund und Ländern verantwortlich sein? - Antworten zum Fragenkatalog für die öffentliche Anhörung zu den Finanzthemen am Freitag, dem 22. Juni 2007, K-Drs. 022 vom 25.05. 2007, 25; Feld, L.P., a. a. O., 7; Huber, B., a. a. O., 3.

21 Fuest, C., a. a. O., 6.

22 Sachverständigenrat zur Begutachtung der gesamtwirtschaftlichen Entwicklung, a. a. O., 3.

23 Wieland, J.: Schriftliche Stellungnahme für die öffentliche Anhörung am 22. Juni 2007, K-Drs. 030 vom 07.06. 2007, 6.

24 Meyer, H., a. a. O., 8.

25 Junkernheinrich, M., a.a.O., 17. 
Die Verschuldung der Länder habe darüber hinaus auch spezifische Ursachen. Zunächst sei dabei die starke nivellierende Wirkung des Finanzausgleichs zu nennen, durch die etwaige Steuermehreinnahmen kaum dem betreffenden Land zugute kämen. ${ }^{26}$ Mithin fehle es an Anreizen für eine nachhaltige Finanzpolitik. ${ }^{27}$ Daneben bestehe auch ein grundsätzliches Missverhältnis zwischen der Höhe der Einnahmen und dem Ausgabenbedarf der Länder und Gemeinden. ${ }^{28}$ Sie hätten in großem Umfang fremdbestimmte Aufgaben zu erfüllen, verfügten aber kaum über Kompetenzen zur Beeinflussung ihrer Einnahmen, so dass sie auf die Möglichkeit zur Verschuldung ausweichen müssten. ${ }^{29}$ Insgesamt habe die kommunale Verschuldung jedoch in deutlich geringerem Maße als die der übrigen Gebietskörperschaften zugenommen. Dies sei vor allem dadurch zu erklären, dass Gemeinden und Kreise durch das jeweilige Landesrecht deutlich strengeren Beschränkungen unterworfen seien. ${ }^{30}$

Unter den von den Sachverständigen unterbreiteten Vorschlägen für eine neue bzw. reformierte „Schuldenbremse“ ist zunächst der eines enger gefassten (Netto-)Investitionsbegriffs zu nennen. Demnach sollten Darlehen für Investitionszwecke und Investitionshilfen nicht unter den Investitionsbegriff fallen, ebenso sei die diskutierte Ausdehnung auf die Bildungsausgaben abzulehnen. ${ }^{31}$ Dadurch würde sich der Verschuldungsspielraum verringern, zudem wäre gesichert, dass der Kreditaufnahme entsprechendes öffentliches Vermögen gegenüberstehe. ${ }^{32}$ Die in diesem Rahmen mögliche Verschuldung wäre nach Ansicht der Befürworter durch das bereits benannte Argument der generationsübergreifenden Lastenverteilung gerechtfertigt. In diesem Zusammenhang müsse jedoch bedacht werden, ob der durch die Investitionen entstehende Nutzen aus Sicht künftiger Generationen die durch die Verschuldung bedingten Kosten aufwiege. Zudem sei der demographische Wandel zu berücksichtigen, aufgrund dessen sich die Lasten auf eine geringer werdende Bevölkerung verteilten ${ }^{33}$, was den individuellen

26 Feld, L.P., a. a. O., 8.

27 Häde, U., a.a.O., 18; Fuest, C., a. a. O., $13 \mathrm{f}$.

28 Hoff, B.I.: Stellungnahme zum Fragenkatalog für die öffentliche Anhörung zu den Finanzthemen am Freitag, dem 22. Juni 2007, der Kommission von Bundestag und Bundesrat zur Modernisierung der Bund-Länder-Finanzbeziehungen. K-Drs. 032 vom 08.06. 2007, 8; Junkernheinrich, M., a. a. O., 14.

29 Korioth, S., a.a.O., 6.

30 Ausführlich Junkernheinrich, M., a. a. O., $8 \mathrm{ff}$.

31 Korioth, S., a.a.O., 7; Blankart, C.B., a.a.O., 27.

32 Ob dies gegenwärtig der Fall ist, wird angezweifelt, vgl. Fuest, C., a. a. O., 8.

33 Blankart, C.B., a.a.O., 46. 
Anteil erhöhe. Schließlich wurde auf die Gefahr verwiesen, dass eine politische Diskussion über eine Neudefinition des mit erheblichen Abgrenzungsproblemen behafteten Investitionsbegriffs auch eine Ausweitung zum Ergebnis haben könne. $^{34}$

Ein zweiter Vorschlag zielt auf die in der bisherigen Regelung enthaltene Ausnahmeklausel für den Fall einer „Störung des gesamtwirtschaftlichen Gleichgewichts“. Auf Länderebene könne diese abgeschafft werden, da das gesamtwirtschaftliche Gleichgewicht ohnehin die Steuerungsfähigkeit eines einzelnen Landes übersteige, eine ihrem Sinn gemäße Anwendung der Klausel mithin kaum vorstellbar sei. ${ }^{35}$ Für die Bundesebene solle sie präziser gefasst werden. ${ }^{36}$ Demgegenüber plädierte der Sachverständige Junkernheinrich für die vollständige Streichung der Ausnahmeklausel. Der Rechtsbegriff des gesamtwirtschaftlichen Gleichgewichts und seiner Störung sei zu unbestimmt und habe keine begrenzende Wirkung. Zudem lasse sich eine erfolgreiche konjunkturpolitische Anwendung in der Vergangenheit kaum nachweisen. ${ }^{37}$

Als Alternative zur bisherigen Kopplung der zulässigen Verschuldung an die Investitionen wurde das Gebot eines mittelfristig (d.h. über einen bestimmten mehrjährigen Zeitraum) ausgeglichenen Haushalts erörtert. Dadurch würden nicht nur die benannten Probleme der Orientierung am Investitionsbegriff vermieden, eine solche Regelung sei auch für die Öffentlichkeit leichter nachvollziehbar. ${ }^{38}$ Vor allem aber sei der Ausgleich von Einnahmen und Ausgaben eine zentrale Voraussetzung für ein funktionierendes Staatswesen und somit als Leitbild für die Finanzverfassung zwingend; zudem sei ein solches Modell kompatibel mit den europäischen Bestimmungen. ${ }^{39}$

In diesem Zusammenhang sei aber auch die unmittelbare Übernahme der Bestimmungen des Europäischen Stabilitäts- und Wachstumspakts (StWP) in Form eines „Nationalen Stabilitätspakt“ zu erwägen. Demnach wären Bund und Länder nicht nur zu mittelfristig ausgeglichenen Haushalten verpflichtet, sie unterlägen auch einer verbindlichen Obergrenze für die jährliche Neuverschuldung in

34 Feld, L.P., a.a.O., 23.

35 Korioth, S., a.a.O., 8.

36 Lenk, T., a. a. O., $10 \mathrm{f}$.

37 Junkernheinrich, M., a. a. O., $23 \mathrm{f}$.

38 Fuest, C., a.a.O., 9.

39 Junkernheinrich, M., a. a. O., 22. 
Höhe von insgesamt $3 \%$ des BIP..$^{40}$ Dieser Spielraum wäre auf die Gebietskörperschaften zu verteilen, wobei sich in vertikaler Hinsicht ein Bundesanteil von $45 \%$ anböte, die horizontale Aufteilung auf die einzelnen Länder auf der Einwohnerzahl und/oder dem BIP basieren sollte. ${ }^{41}$ Hierdurch entfiele der bisherige Dualismus von europäischem und deutschem Recht, ${ }^{42}$ zudem habe sich trotz aller Kritik der StWP im Vergleich zur deutschen Verschuldungsregel als weitaus wirksamer erwiesen. ${ }^{43}$ Allerdings sei nicht nur zu bedenken, dass Deutschland die europäische Defizitregel in vier aufeinander folgenden Jahren nicht einhalten konnte, sondern auch die Schwierigkeit, in einem Nationalen Stabilitätspakt vorzusehende Sanktionsmechanismen politisch durchzusetzen. ${ }^{44}$

Die Vertreter des Sachverständigenrates zur Begutachtung der gesamtwirtschaftlichen Entwicklung schließlich verwiesen auf ihren im Auftrag des Bundeswirtschaftsministeriums erarbeiteten Vorschlag, der aus drei „Modulen " besteht. ${ }^{45}$ Das erste Modul beinhaltet die langfristige Verschuldungsbegrenzung. Demnach sei der Investitionsbegriff des Art. 115 GG dahingehend zu modifizieren, dass die langfristige Nettokreditaufnahme die Nettoinvestitionen nicht übersteigen dürfe. Die Nettoinvestitionen wiederum seien zu definieren als die Summe der gesamten Investitionsausgaben abzüglich der Investitionseinnahmen und der kalkulatorischen Abschreibungen.

Das zweite Modul soll die kurzfristige Verschuldung begrenzen, zum einen durch eine Ausgabenregel, zum anderen durch ein Ausgleichskonto; die benannte Ausnahmeklausel des Art. 115 GG sollte entfallen. Nach der Ausgabenregel dürften die Ausgaben einer Gebietskörperschaft (abzüglich der gemäß Modul 1 kreditfinanzierten Nettoinvestitionen) die konjunkturbereinigten Einnahmen nicht übersteigen. Die Konjunkturbereinigung erfolgte durch die Multiplikation der tatsächlichen Einnahmen mit einem Konjunkturfaktor, der sich wiederum als

Dies ginge über den im Rahmen der „Föderalismusreform I“ eingefügten Art. 104a Abs. 6 GG hinaus, der ebenfalls gelegentlich als „Nationaler Stabilitätspakt“ bezeichnet wird. Er regelt jedoch nur die Verteilung durch die Europäische Union verhängter Strafzahlungen auf Bund und Länder.

41 Fuest, C., a.a.O., 10 f; Huber, B., a. a.O., 4.

42 Huber, B., a. a. O., 4.

43 Sachverständigenrat, a.a. O., 3.

44 Huber, B., a. a. O., 4.

45 Sachverständigenrat, a.a.O., $3 \mathrm{ff}$. Ausführliche Darstellung in: ders.: Staatsverschuldung wirksam begrenzen - Expertise im Auftrag des Bundesministers für Wirtschaft und Technologie, Wiesbaden, März 2007. Ähnlich aufgebaut ist die sog. ,Schweizer Schuldenbremse“, auf die hier nicht näher eingegangen wird. Vgl. dazu ausführlich Müller, C.: Aspekte einer Schuldenbegrenzung nach helvetischem Vorbild, K-Drs. 028 vom 25.05. 2007. 
Anteil des Produktionspotenzials ${ }^{46}$ am BIP berechnen soll. Abweichungen von dieser Regel wären nur in begründeten Ausnahmefällen durch mit Zweidrittelmehrheit gefassten Bundestagsbeschluss möglich. Auf dem Ausgleichskonto würden die in der Haushaltsrechnung festgestellten tatsächlichen Abweichungen von den zulässigen Verschuldungsspielräumen verbucht; ab dem Überschreiten einer festzulegenden Grenze müssten diese abgebaut werden.

Als drittes Modul solle ein verfassungsrechtlich abgesicherter Sanktionsmechanismus vorgesehen werden, der im Extremfall (Überschreitung des Ausgleichskontos in zwei aufeinander folgenden Jahren) für den Bund die Erhebung eines Zuschlags auf die Einkommensteuerschuld mit Bindung an die Rückführung des Ausgleichkontos vorsieht, für die Länder die entsprechende Bindung eines Teils des einwohnerbezogenen Umsatzsteuervorwegausgleichs.

Gegen den Vorschlag des Sachverständigenrates wurde zum einen die Tatsache angeführt, dass kein Konsens über die Bestimmung des zur Berechnung des Konjunkturfaktors erforderlichen Produktionspotenzials bestehe, ${ }^{47}$ und zum anderen darauf verwiesen, dass die Dualität nationaler und europäischer Regelungen zur Defizitbegrenzung dann fortbestünde. ${ }^{48}$

Zur Begrenzung der kommunalen Verschuldung wurden schließlich mehrere Einzelmaßnahmen vorgeschlagen:

- die Übertragung der für Bund und Länder getroffenen Neureglungen auf die Kommunen durch die Länder; ${ }^{49}$

- die Berücksichtigung aller ausgelagerten Bereiche bei der Prüfung durch die Kommunalaufsicht; ${ }^{50}$

- verschärfte Regelungen zur Aufnahme von Kassenkrediten. ${ }^{51}$

46 Unter dem Produktionspotenzial wird die Produktionsleistung verstanden, die von einer Volkswirtschaft in einer Periode bei Voll- bzw. Normalauslastung der Kapazitäten erbracht werden kann.

47 Häde, U., a. a. O., 11.

48 Huber, B., a.a. O., 5.

49 Seitz, H., a. a.O., 59.

50 Junkernheinrich, M., a. a. O., 9.

51 Blankart, C.B., a.a. O., 42; Lenk, T.: Stellungnahme zum Fragenkatalog der Föderalismuskommission II, K-Drs. 026, 12. 


\section{Weitere Instrumente zur Bewältigung und Verhinderung von Haushaltskrisen}

Das Bundesverfassungsgericht hatte in seinem „Berlin-Urteil“ im Oktober 2006 klargestellt, dass überschuldete Länder für den Abbau der Verschuldung prinzipiell selbst verantwortlich sind. Anspruch auf Sanierungshilfen des Bundes oder der übrigen Länder besteht nur für den Fall, dass alle übrigen Möglichkeiten ausgeschöpft sind. ${ }^{52}$ Vor diesem Hintergrund war auch der Umgang mit der hohen Verschuldung einiger Länder Thema der Beratungen.

Von Seiten mehrer Sachverständiger wurde das Prinzip der Schuldnerselbstverantwortung betont. ${ }^{53}$ Demnach wäre jede Gebietskörperschaft für ihre Schulden und deren Abtragung und könnte nicht auf Hilfen der bundesstaatlichen Solidargemeinschaft hoffen. Durch eine solche Klarstellung würde der Markt eine überhöhte Verschuldung eines Landes durch einen erhöhten Zinssatz sanktionieren und so die Kreditaufnahme insgesamt begrenzen. Für eine konsequente Umsetzung dieses Systems wäre allerdings auch ein erweiterter Spielraum der Länder auf der Einnahmenseite in Form von Steuerautonomie erforderlich. Da dieses System aber die Kreditkosten für die öffentliche Hand insgesamt erhöhen würde, wäre es nach Ansicht des Sachverständigen Meyer nur dann sinnvoll, wenn verfassungsrechtliche Regelungen zur Verschuldungsbegrenzung weniger wirksam oder mit anderen Nachteilen verbunden wären. ${ }^{54}$

Auch ohne die Einführung einer Schuldnerselbstverantwortung könnte eine erweiterte Steuerautonomie für die Länder zur Abtragung von Schulden sinnvoll sein. Der Sachverständige Korioth etwa schlug vor, Ländern, die sich in einer Verschuldungskrise befänden, die Möglichkeit zur Erhebung von Zuschlägen in Höhe von bis zu 15 \% auf die Einkommen- und Körperschaftsteuer zu gewähren. Hinzu käme die Befugnis zur Gesetzgebung über die nach Art. 106 Abs. 2 GG den Ländern zustehenden Steuern. Diese Möglichkeit müsse allerdings mit Automatismen verbunden sein, die strategisches Verhalten ausschlössen; zudem sei das betroffene Land auf die Nutzung sämtlicher Konsolidierungspotenziale auf der Ausgabenseite zu verpflichten. Schließlich sei zu bedenken, dass dieses Ver-

53 Ausführlich Blankart, C. B., a. a. O.; außerdem etwa Feld, L.P., a. a. O., $11 \mathrm{f}$.

54 Meyer, H., a.a.O., 17. 
fahren durch eventuelle „Abwanderungseffekte“ das ökonomische und fiskalische Gefälle zwischen den Ländern vergrößern könne. ${ }^{55}$

Schließlich wurde auch die Einrichtung eines Entschuldungsfonds von mehreren Sachverständigen erörtert. Darin könnten die bis zu einem Stichtag aufgelaufenen Schulden mehrer oder aller Gebietskörperschaften übertragen werden, die Zinsbelastungen würden entweder durch den Bund oder durch Bund und Länder nach Leistungsfähigkeit getragen. ${ }^{56}$ Er könne somit „einen substantiellen Beitrag zur Schaffung gleichwertiger Rahmenbedingungen für Landespolitik und zur nachhaltigen Haushaltskonsolidierung leisten “57. Der Vorschlag wurde jedoch überwiegend abgelehnt, da ein solcher Fonds unsolide Haushaltspolitik nachträglich belohnen würde und somit negative Anreizwirkungen hätte. ${ }^{58}$

\section{Erweiterte Steuerautonomie für die Länder}

Wie angesprochen, traten die zentralen Charakteristika der föderalstaatlichen Finanzverfassung, Steuerverbund und Finanzausgleich, in der Diskussion hinter den Komplex der Verschuldungsbegrenzung und -rückführung zurück. Dennoch war auch das Steuersystem Thema der Sachverständigenanhörung, zumal - wie etwa bei der Frage des Umgangs mit hoch verschuldeten Ländern deutlich wurde - inhaltliche Zusammenhänge bestehen. Im Wesentlichen konzentrierten sich die eingebrachten Vorschläge auf regionale $\mathrm{Zu}$ - und Abschlags- bzw. Hebesatzrechte. Die Bemessungsgrundlagen sollten hingegen weiter bundeseinheitlich geregelt werden.

Blickt man zunächst auf die Gemeinschaftssteuern (Einkommen-, Körperschaftund Umsatzsteuer), deren Aufkommen Bund und Ländern anteilig zufließt, so gilt die Umsatzsteuer aufgrund der Besonderheiten bei ihrer Erhebung allgemein als ungeeignet für die Einräumung von Länderkompetenzen. ${ }^{59}$ Für Hebesatzrechte der Länder kommt nach Ansicht der Sachverständigen am ehesten die Einkommensteuer in Frage, da die reformierte Steuer auch vom Aufkommen her in der Lage sein sollte, einen substanziellen Beitrag zur Verbesserung der Länderfi-

55 Korioth, S., a.a.O., $11 \mathrm{f}$.

56 Korioth, S., a. a. O., 10.

57 Renzsch, $W$. , a. a. O., 7.

58 Huber, B., a.a.O., 9 f.; Fuest, C., a. a.O., 20; Korioth, S., a.a.O., 11.

59 Korioth, S., a.a. O., 17. 
nanzen zu leisten. ${ }^{60}$ Die Vorschläge gehen dahin, die Bemessungsgrundlage und den Steuertarif weiter bundeseinheitlich zu regeln, den Ländern jedoch die Möglichkeit einzuräumen, innerhalb eines Korridors (etwa $3 \%{ }^{61}$ oder $5 \%{ }^{62}$ des Steuersatzes) variable $\mathrm{Zu}$ - und Abschläge zu erheben.

Bei der Körperschaftsteuer kämen grundsätzlich ähnliche länderspezifische $\mathrm{Zu}$ und Abschlagsrechte in Betracht. ${ }^{63}$ Es könnten sich jedoch insofern Nachteile für Betriebe mit nur einem Standort ergeben, als sich Unternehmen mit mehreren Standorten erhebliche Gestaltungsspielräume eröffneten. ${ }^{64}$

Für eine Ausweitung regionaler Steuerautonomie kämen prinzipiell alle Ländersteuern in Betracht, für die die Gesetzgebungskompetenz derzeit beim Bund liegt. Nach Ansicht des Sachverständigen Seitz liege „,[v]on der Sache her [...] nur eine Lösung nahe. Über die Steuern, deren Ertrag den Ländern allein zukommt, sollten sie die ausschließliche Gesetzgebungskompetenz haben." ${ }^{65}$ Aufgrund des geringen Aufkommens dieser Steuern wird eine solche Maßnahme allerdings nicht als ausreichend für eine bessere finanzielle Ausstattung der Länder angesehen. ${ }^{66}$

Mit Blick auf die Folgen einer solchen Umgestaltung des Steuersystems gaben die Sachverständigen unterschiedliche Prognosen ab. So fördere Steuerautonomie nach Ansicht von Feld den Steuerwettbewerb unter den Ländern, sorge so für einen sparsamen Umgang mit knappen Mitteln und einen „pfleglicheren Umgang" mit der eigenen Steuerbasis. ${ }^{67}$ Dagegen wurde von Konrad eingewandt, dass nur finanzstarke Länder in der Lage wären, Abschläge zu gewähren; folglich käme es zu Anpassungshandlungen wie Verlagerungen von Unternehmens- und Wohnsitzen. Daher seien auf regionaler Ebene nur Steuerkompetenzen für relativ immobile Bemessungsgrundlagen anzusiedeln. ${ }^{68}$ Renzsch vertrat hierzu die Ansicht, dass die Transaktionskosten von Wanderungen unterschätzt würden. Faktoren wie Arbeitsplatz, Lebensqualität und Grundstücks- bzw. Woh-

Vgl. Fuest, C., a.a. O., 19; Kirchgässner, G., a.a. O., 2 FN 3.

61 Renzsch, $W .$, a.a.O., 22.

62 Korioth, S., a.a.O., 17.

63 Huber, B., a. a. O., 7f.

64 Seitz, H., a.a.O., 108.

65 Meyer, H., a.a.O., 20.

66 Kirchgässner, G., a.a. O., 2.

67 Feld, L.P., a.a.O., 39.

68 Konrad, K. A. , a. a. O., 27. 
nungspreisen käme größere Bedeutung als der steuerlichen Belastung zu. ${ }^{69}$ Laut Wieland missachteten länderspezifische $\mathrm{Zu}$ - und Abschlagsrechte die Eigenart der Einkommen- und Körperschaftsteuer, die ebenso wie die Umsatzsteuer Steuern der bundesstaatlichen Gemeinschaft und nicht ihrer einzelnen Glieder seien. Sie spiegelten die Wirtschaftskraft Deutschlands insgesamt wider und ließen sich nicht einzelnen Ländern zuordnen, denen deshalb auch keine autonomen Steuererhebungsrechte eingeräumt werden könnten. ${ }^{70}$ Der häufig geäußerten Befürchtung, ausländische Investoren könnten durch von Land zu Land unterschiedliche Regelungen und die damit einhergehende weitere Verkomplizierung des deutschen Steuerrechts abgeschreckt werden, widersprach der Sachverständige Kirchgässner. Die Erfahrungen anderer föderaler Staaten wie etwa der Schweiz zeigten, dass dies kein zwingender Effekt sei. ${ }^{71}$

Hinzu komme die Wirkung grenzüberschreitender Effekte. So stehe eine steuerliche Dezentralisierung in Deutschland im Widerspruch zu den Vereinheitlichungsbemühungen auf europäischer Ebene. ${ }^{72}$ Andererseits sei zu berücksichtigen, dass Deutschland sich insgesamt im internationalen Steuerwettbewerb befinde, eine vollkommene steuerliche Harmonisierung auf EU-Ebene aber unwahrscheinlich sei. Es komme darauf an, wie Deutschland sich in diesem Wettbewerb positioniere. Unterschiedliche Steuersätze in den Ländern könnten in diesem Zusammenhang einen Vorteil für Deutschland bringen. ${ }^{73}$ Schließlich wären Folgeänderungen im Länderfinanzausgleich nötig, da Einnahmenunterschiede, die auf die Nutzung der Steuerautonomie zurückzuführen seien, außer Betracht bleiben müssten. Hierzu wäre die Bemessungsgrundlage des Länderfinanzausgleichs um die unter die Ländergesetzgebung fallenden Steuern zu bereinigen. $^{74}$

\section{Die politische Diskussion}

In der politischen Diskussion zu den benannten Problemkomplexen fällt erneut das bereits benannte Ungleichgewicht auf: Der klare Schwerpunkt liegt auf ei-

69 Renzsch, W., a. a. O., 21.

70 Wieland, J., a. a. O., 15.

71 Kirchgässner, G., a.a.O., $16 \mathrm{f}$.

72 Konrad, K.A., a.a.O., 27.

73 Kirchgässner, G., a.a.O., 17.

74 Korioth, S., a.a.O., 14-15. 
nem möglichen gemeinsamen Regelwerk zur Verhinderung von Haushaltskrisen und zum Abbau der aufgelaufenen Verschuldung der Gebietskörperschaften. Der Kern der Finanzverflechtung zwischen Bund und Ländern, also der Steuerverbund und der Finanzausgleich, tritt demgegenüber in den Hintergrund. Diese Fokussierung deckt sich mit der dem Einsetzungsbeschluss der Kommission beigefügten „offenen Themensammlung“, die mit Blick auf die Haushalts- und Schuldenproblematik die bei weitem größte Ausdifferenzierung aufweist. ${ }^{75}$

Bereits vor der Einsetzung der Kommission hatte sich etwa der niedersächsische Ministerpräsident Wulff (CDU) für einen „nationalen Entschuldungsplan“ ausgesprochen. ${ }^{76}$ Demnach sei die aufgelaufene Verschuldung der Gebietskörperschaft nur durch gesamtstaatlich koordinierte Maßnahmen zu bewältigen. Bund und Länder sollten sich verpflichten, ihre Haushalte zu konsolidieren und ab einem festzulegenden Zeitpunkt keine neuen Schulden mehr aufzunehmen; fortan wäre dies nur noch im Ausnahmefall durch einen mit Zweidrittelmehrheit gefassten Parlamentsbeschluss möglich. Die aufgelaufenen Altschulden wären einem ,nationalen Entschuldungsfonds“ zu übertragen und nach Leistungsfähigkeit abzutragen.

Eine ähnliche Stoßrichtung wiesen die meisten der im Rahmen der Kommissionsberatungen von den politischen Akteuren vorgebrachten Reformkonzepte auf. Dabei ging es vor allem um eine Verknüpfung der Regelungen zur Begrenzung der Kreditaufnahme durch die öffentliche Hand und des Umgangs mit den Altschulden. Einerseits sollten sich die Gebietskörperschaften auf wirkungsvolle Maßnahmen zur Begrenzung der Neuverschuldung einigen, andererseits wäre den finanzschwachen Ländern Unterstützung beim Abtragen der aufgelaufenen Schulden zu gewähren.

So wurde zu Beginn der Kommissionsarbeiten die grundsätzliche Zielrichtung eines Abbaus der Staatsverschuldung und der Vermeidung von Haushaltsnotlagen in einem überparteilichen Diskussionspapier der in der Kommission vertretenen Mitglieder der Bundesregierung deutlich, freilich ohne dies mit konkreten Handlungsoptionen zu verbinden. ${ }^{77}$ Stärker ins Detail gingen hingegen die zur selben Zeit publizierten Leitlinien der Arbeitsgruppe der Unionsfraktion im Bundestag. So sollte eine Nettokreditaufnahme künftig nur noch in Ausnahmefällen

75 Deutscher Bundestag, 16. Wahlperiode, a. a. O., Anlage 1; Bundesrat, a.a. O., Anlage 1.

76 Wulff, C.: Wir brauchen einen nationalen Entschuldungsplan, in: ZSE, 4/2 (2006), 181-187.

77 Mitglieder des Bundesregierung in der Kommission: Diskussionspapier, K-Drs. 005 vom 12.03. 2007. 
unter der Voraussetzung eines verbindlichen Tilgungsplans zulässig sein, zudem wurde eine gesetzliche Verpflichtung zum Schuldenabbau in Wachstumszeiten verlangt. Ebenfalls enthalten waren die Forderungen nach verstärkten Leistungsanreizen im Länderfinanzausgleich, erweiterter Steuerautonomie der Länder sowie einer insgesamt weniger verflochtenen Finanzverfassung. ${ }^{78}$

Darüber hinaus vertrat in der Anfangsphase nur die FDP die Forderung eines grundsätzlichen Verbots von Nettoneuverschuldung, deren Parteitagsbeschluss vom Juni 2007 zudem ebenfalls erweiterte Steuerkompetenzen der Länder und eine anreizorientierte Reform des Finanzausgleichs forderte, sich den Vorschlag eines „Entschuldungsfonds“ aber nicht zu eigen machte. ${ }^{79}$ Die Grünen wiederum lehnten ein generelles Verschuldungsverbot ab; ihrer Ansicht nach sollten die Haushalte lediglich über einen Konjunkturzyklus hinweg ausgeglichen sein. Einer Reform des Steuerverbundes standen auch sie grundsätzlich positiv gegenüber. $^{80}$

Im weiteren Verlauf der Kommissionsarbeiten traten zunächst vor allem Mitglieder von Landesregierungen mit eigenen Konzepten an die Öffentlichkeit. Besondere öffentliche Aufmerksamkeit erfuhr etwa der baden-württembergische Ministerpräsident und Kommissionsvorsitzende Oettinger (CDU), der sich dafür aussprach, finanzschwachen Ländern Beihilfen zur Schuldentilgung zu gewähren. Zu den Mittel, die diese aus eigener Kraft aufwendeten, sollte ein Zuschlag von $20 \%$ bis $100 \%$ aus der Solidargemeinschaft gewährt werden. Sanktionsbewehrte „Schuldenbremsen“ zur Reduzierung der Neuverschuldung lehnte Oettinger hingegen $\mathrm{ab}^{81}$

Weiter ging der schleswig-holsteinische Regierungschef Carstensen (CDU), der - ähnlich wie zuvor sein Amtskollege Wulff - die Übertragung der Altschulden in einen gemeinschaftlichen Fonds forderte. Zur Begrenzung der Neuver-

78 AG Föderalismus II der CDU/CSU-Bundestagsfraktion: Leitlinien, K-Drs. 003, ohne Datum.

79 Bundesparteitag der FDP (Stuttgart, 15.-17.06. 2007): Modernisierung der Bund-Länder-Finanzbeziehungen, K-Drs. 039; ähnlich FDP-Bundestagsfraktion: Ausstieg aus dem Schuldenstaat - Einstieg in mehr Generationengerechtigkeit, K-Drs. 094 vom 12.02. 2008.

80 Grüne AG zur Föderalismuskommission II: 10 Thesen zur Modernisierung der Bund-Länder-Finanzbeziehungen, K-Drs. 037, ohne Datum; siehe auch die Position des Vorsitzenden der grünen Landtagsfraktion in Baden-Württemberg, Kretschmann, W.: Föderalismusreform II, K-Drs. 036 vom 17.04. 2007.

81 Oettinger, G.: Wer Schulden tilgt, muss belohnt werden, Interview in: Die Welt vom 22. 05. 2007. 
schuldung warb er für an die Maastricht-Kriterien angelehnte Restriktionen. ${ }^{82}$ Der rheinland-pfälzische Finanzminister Deubel (SPD) hingegen schlug einen Entschuldungsfonds nur für hoch verschuldete Länder vor, der Zahlungen nur bei entsprechenden Eigenanstrengungen leisten sollte. Statt einer stärkeren Begrenzung der Neuverschuldung sprach er sich für eine Übernahme des in Rheinland-Pfalz auf kommunaler Ebene angewendeten Stabilisierungsfonds aus. Nach diesem Modell fließt in Wachstumsphasen automatisch ein Teil der Steuereinnahmen in den genannten Fonds, der in Krisenjahren den öffentlichen Haushalten zur Verfügung steht. ${ }^{83}$

Seitens der Bundesregierung stellte Finanzminister Steinbrück (SPD) im Februar 2008 ein Modell für eine modifizierte „Schuldenbremse“ vor. Im Zentrum steht dabei eine Differenzierung zwischen konjunktureller und struktureller Neuverschuldung. Letztere umfasst die um konjunkturelle Effekte bereinigte Nettokreditaufnahme, die für alle öffentlichen Haushalte insgesamt auf 0,5\% des BIP zu begrenzen wäre. Von diesem Verschuldungsspielraum würde der Bund 0,35 \% des BIP in Anspruch nehmen. In Jahren wirtschaftlichen Wachstums würde die zulässige Verschuldung entsprechend sinken, in Krisenjahren steigen. Eine über das zulässige Maß hinausgehende Nettokreditaufnahme wäre nur noch in Notsituationen (etwa Naturkatastrophen) unter der Voraussetzung eines mit qualifizierter Mehrheit gefassten Parlamentsbeschlusses möglich. Zur Problematik der Altschulden der Länder äußerte sich der Vorschlag nicht. ${ }^{84}$

Kurz darauf veröffentlichte die CDU/CSU-Bundestagsfraktion ein Diskussionspapier, das in dieselbe Richtung ging. Als wesentlicher Unterschied zum dargelegten Vorschlag des Bundesfinanzministers stach dabei die Forderung heraus, die strukturelle Verschuldung auf null zu reduzieren, also Neuverschuldung nur noch im Rahmen des konjunkturellen Ausgleichs zuzulassen. Eine Beteiligung des Bundes am Abbau der Altschulden der Länder wurde ausdrücklich abgelehnt. $^{85}$

Carstensen, P. H.: Thesen zur MBO II, K-Drs. 041 vom 30.06. 2007; bekräftigt durch ders.: Schreiben an die Vorsitzenden der Kommission von Bundestag und Bundesrat zur Modernisierung der BundLänder-Finanzbeziehungen, K-Drs. 106 vom 23.04. 2008.

83 Deubel, I.: Der Stabilisierungsfonds: Ausgleich konjunktureller Schwankungen, Abbau struktureller Defizite und Bewältigung von Haushaltskrisen, K-Drs. 056 vom 13./14.09. 2007.

84 Bundesministerium der Finanzen: Notwendigkeit und Inhalt einer neuen Schuldenregelung im Grundgesetz, K-Drs. 096 vom 25.02. 2008. 
Hingegen legte jüngst der hessische Ministerpräsident Koch (CDU) einen Vorschlag vor, der erneut ein strenges Verschuldungsverbot und eine Lösung für die Altschulden miteinander verknüpft. Demnach wäre ein generelles Verbot der Nettokreditaufnahme für alle Gebietskörperschaften bis 2012 bzw. 2015 schrittweise einzuführen, Ausnahmeregelungen sollten lediglich für Naturkatastrophen oder einen extremen Einbruch der Steuereinnahmen gelten. Im Gegenzug würden alle bislang aufgelaufenen öffentlichen Schulden auf einen gemeinsamen Fonds überführt und aus diesem innerhalb von 50 Jahren abgetragen werden. Zwar wären die Beiträge zu diesem Fonds grundsätzlich nach dem Verursacherprinzip zu bemessen, doch sollten für besonders finanzschwache Länder Beihilfen vorgesehen werden. ${ }^{86}$

Gegenüber diesen Vorschlägen zum Umgang mit der Schuldenproblematik, die durchaus eine gemeinsame Stoßrichtung und mögliche Kompromisslinien erkennen lassen, finden sich nur punktuelle Äußerungen zu einer Reform des Steuerverbundes und des Finanzaugleichs. $\mathrm{Zu}$ nennen wären zunächst die bereits erwähnten Positionen von Grünen und FDP. Hinzu kommen einzelne Vorschläge von Ländervertretern.

So legte der saarländische Ministerpräsident Müller (CDU) ein im Auftrag der Landesregierung durch das Institut der deutschen Wirtschaft erarbeitetes „Optionsmodell“ zur begrenzten Reform von Steuerverbund und Finanzausgleich vor. Demnach sollte finanzschwachen Ländern für eine begrenzte Zeit die Möglichkeit eingeräumt werden, über eine veränderte Berechnungsmethode im Finanzausgleich unmittelbar von einer überdurchschnittlichen Entwicklung des örtlichen Steueraufkommens zu profitieren; dies wäre jedoch verbunden mit einem entsprechenden Verlustrisiko bei unterdurchschnittlicher Entwicklung. ${ }^{87}$

Der Berliner Finanzsenator Sarrazin (SPD) schlug eine grundlegende Reform des Steuerverbundes vor, die insbesondere eine Abkehr vom örtlichen Aufkommensprinzip beinhaltete. Gesetzgebung und Verwaltung der Gemeinschaftssteuern sollten demnach allein beim Bund liegen. Der Anteil der Länder wäre nicht mehr an das tatsächliche Steueraufkommen gekoppelt, sondern an die konjunktu-

86 Koch, R.: Hessisches Modell zur Lösung des Verschuldungsproblems. Aufgabenfinanzierung auf Kosten zukünftiger Generationen beenden - Generationenvertrag für einen Weg aus der Schuldenfalle-, K-Drs. 107 vom 23.04. 2008; vgl. auch ders.: Ein Generationenvertrag über den Ausweg aus der Schuldenfalle, in: FAZ vom 26.04. 2008.

87 Staatskanzlei des Saarlandes: Wege aus der Haushaltsnotlage - Das Optionsmodell als Beitrag zur Reform der föderalen Finanzbeziehungen (Föderalismusreform II), K-Drs. 025 vom 24. 05. 2007. 
relle Entwicklung und entsprechend als Prozentzahl des BIP festzulegen. Die Verteilung dieses Anteils auf die einzelnen Länder sollte zu $75 \%$ nach der Einwohnerzahl erfolgen, zu $25 \%$ leistungsbezogen nach der regionalen Wirtschaftskraft. ${ }^{88}$ Schließlich wiesen die Vertreter der Landtage wiederholt auf eine ihrer Ansicht nach notwendige Verknüpfung einer restriktiveren (und damit das parlamentarische Budgetrecht einschränkenden), Schuldenbremse“ mit einer im Gegenzug erweiterten Einnahmenautonomie der Länder durch entsprechende Steuergesetzgebungskompetenzen hin. ${ }^{89}$

\section{Ausblick}

Insgesamt zeigt sich, dass eine politische Einigung auf Reformen im Bereich der „Finanzthemen“ am ehesten mit Blick auf die Verschuldungsproblematik erreichbar sein dürfte. Hier zeichnet sich eine mögliche Kompromisslinie insofern $a b$, als eine Zustimmung auch finanzschwächerer Länder zu einer restriktiveren Regelung der staatlichen Kreditaufnahme über eine Gewährung von Beihilfen zur Tilgung der Altschulden erreichbar sein könnte. Demgegenüber ist das Interesse an einer Reform des Steuerverbunds und des Finanzausgleichs in der Kommission offenbar deutlich geringer. Die wenigen dazu von Seiten der politischen Akteure vorgebrachten Vorschläge gehen weit auseinander und lassen bislang keine Möglichkeit für eine Einigung erkennen.

\section{ZSE-Redaktion unter Mitarbeit von Volker Reiprich und Simon Schubert}

Sarrazin, T.: Systematisierung und Vereinfachung der Finanzverteilung zwischen Bund und Ländern sowie der Steuergesetzgebung und -verwaltung. Überlegungen zur Neuordnung der Artikel 105-108 GG, K-Drs. 046 vom 10.09. 2007.

Konferenz der Präsidentinnen und Präsidenten der deutschen Landesparlamente: Berliner Erklärung. Bundesstaatliche Finanzbeziehungen modernisieren - Gestaltungsföderalismus statt Beteiligungsföderalismus, K-Drs. 048 vom 31.08. 2007; Kretschmann, W.: Nur eine Lösung im Paket hat eine Chance. Vorschläge zur Begrenzung der Neuverschuldung, Finanzierung eines Altschuldenfonds, Steuerautonomie der Länder und Neuordnung des Länderfinanzausgleichs, K-Drs. 091, Januar 2008; Kayenburg, M. u. a.: Gemeinsame Position der Vertreter der Landtage. Verschuldung begrenzen, Altschulden bewältigen, Finanzausstattung aufgabengerecht gestalten - Landtage stärken!, K-Drs. 092 vom 15.01. 2008; Kayenburg, M. u. a.: Offener Brief der Vertreter der Landtage und der unterzeichnenden Stellvertreter in der Föderalismuskommission II, K-Drs. 100 vom 01.04. 2008. 